\title{
Performer Effective Date Range
}

National Cancer Institute

\section{Source}

National Cancer Institute. Performer Effective Date Range. NCI Thesaurus. Code C93981.

The date and time span for when the performer is active. 